\title{
Constructions Of Traveling Wave Solutions Of The Fractional Nonlinear Model Of The Low-Pass Electrical Transmission Lines
}

\author{
Yeşim SAĞLAM ÖZKAN ${ }^{1}$ \\ ${ }^{1}$ Bursa Uludağ Üniversitesi, Fen Edebiyat Fakültesi, Matematik Bölümü, Bursa. \\ e-posta:ysaglam@uludag.edu.tr ORCID ID: https://orcid.org/0000-0002-1364-5137 \\ Geliş Tarihi: 14.01.2021 Kabul Tarihi: 02.04.2021
}

\begin{tabular}{|c|c|}
\hline & Abstract \\
\hline $\begin{array}{l}\text { Atangana-Baleanu } \\
\text { derivative operator; } \\
\text { improved } \tan (\varphi / 2) \text { - } \\
\text { expansion method; } \\
\text { simplest equation }\end{array}$ & $\begin{array}{l}\text { In this article, the structure of the improved } \tan (\varphi / 2) \text {-expansion method and the simplest equation } \\
\text { method are applied. The fractional nonlinear model of the low-pass electrical transmission lines via } \\
\text { Atangana-Baleanu derivative operator is taken into consideration and exact solutions have been } \\
\text { constructed of this equation using proposed methods. This article explores the applicability and } \\
\text { effectiveness of these methods on fractional nonlinear evolution equations. }\end{array}$ \\
\hline
\end{tabular}

\section{Alçak Geçiren Elektrik İletim Hatlarının Kesirli Mertebeden Lineer Olmayan Modelinin İlerleyen Dalga Çözümlerinin Oluşturulması}

\author{
Öz \\ Anahtar kelimeler \\ Bu makalede, geliştirilmiş $\tan (\varphi / 2)$-açılım yöntemi ve en basit denklem yöntemi uygulanmıştır. Alçak \\ Atangana-Baleanu \\ türev operatörü; \\ geliştirilmiş $\tan (\varphi / 2)$ - \\ açılım yöntemi ; en \\ geçiren elektrik iletim hatlarının Atangana-Baleanu türev operatörü aracılığıyla kesirli mertebeden \\ lineer olmayan modeli dikkate alınmış ve önerilen yöntemler kullanılarak bu denklemin tam çözümleri \\ oluşturulmuştur. Bu makale, bu yöntemlerin kesirli doğrusal olmayan evrim denklemleri üzerindeki \\ uygulanabilirliğini ve etkinliğini araştırmaktadır.
}

basit denklem yöntemi

\section{Introduction}

Fractional nonlinear evolution equation is one of the branches of science that has attracted attention especially in recent years. Fractional analysis studies that started with the discussion between L'Hospital and Leibniz have attracted the attention of researchers for many years. In addition to mathematics, it has a very deep physical application area where it can formulate many different phenomena in different fields such as physics, engineering sciences, economics, chemistry, signal processing, rheology, diffusion processes, food supplements, semi-chaotic dynamic systems, mechanics-mechatronics, seismology, hydrodynamics. Due to its wide scope and diverse applications in different disciplines, the importance of exact (analytical) and numerical solutions of fractional differential equations has increased. Many methods such as the direct algebraic method (Rezazadeh et al. 2017), the sinh-Gordon function method (Yokuş et al. 2020), the decomposition method (Ray 2006), the discrete homotopy perturbation method (Özpınar 2020), the finite forward difference method (Yokuş 2020), the modified homotopy analysis transform method 
(Morales-Delgado et al. 2018), the $\left(m+1 / G^{\prime}\right)$ expansion method (Durur et al. 2020), the subequation method (Tasbozan et al. 2019, Yokuş et al. 2020), the $\left(G^{\prime} / G\right)$ method (Durur 2020, Shang and Zheng 2013), fractional sub-equation method (Yaşar and Yıldırım 2018), (1/G')-expansion method (Durur ve Yokuş 2019, Durur ve Yokuş 2020, Yokuş et al. 2020, Yokuş et al. 2020), the generalization exponential rational method (Khater et al. 2020), the modified auxiliary equation method (Alderremy et al. 2019) etc. to be used to reach solutions have been proposed.

The most popular definitions in the fractional mathematics world are the Riemann-Liouville, Grünwald-Letnikov and Caputo (Podlubny 1999, Caputo 1967, Caputo and Fabrizio 2015) definitions. Atangana-Baleanu (Atangana and Baleanu 2016) fractional derivative and integral due to Caputo and Riemann-Liouville fractional derivatives have played an important role in mathematical modeling these days.

In this study, we construct the solutions for the fractional nonlinear model of the low-pass electrical transmission lines which is given by (Abdou and Soliman 2018)

$$
\begin{gathered}
\mathcal{D}_{t t}^{2 v} W-\alpha \mathcal{D}_{t t}^{2 v} W^{2}+\sigma \mathcal{D}_{t t}^{2 v} W^{3}- \\
\lambda^{2} \mathcal{D}_{x x}^{2 v} W-\frac{\lambda^{4}}{12} \mathcal{D}_{x x x x}^{4 v} W=0,
\end{gathered}
$$

where $W=W(x, t)$ is the function that is used to describe the dynamical behavior (the voltage) of the nonlinear wave processes low-pass electrical transmission lines. Additionally, $\alpha, \lambda, \sigma$ are arbitrary constants while $0<v<1$. The variable $\mathrm{x}$ is interpreted as the propagation distance and $t$ is the slow time. The physical details of the derivation of Eq. (1) using the Kirchhoffs laws are given in (Abdou and Soliman 2018).

Applying the following definition of ABR fractional operator (Atangana and Gomez-Aguilar 2018) to Eq. (1);

Definition 1 It is given by (Fernandez et al. 2019)

$$
\begin{array}{r}
\mathrm{ABR}_{\mathcal{D}_{a+}^{v} \mathcal{F}(t)}= \\
\frac{\mathcal{B}(v)}{1-v} \frac{d}{d t} \int_{a}^{t} \mathcal{F}(x) \mathcal{G}_{v}\left(\frac{-v(\mathrm{t}-v)^{v}}{1-v}\right) d x
\end{array}
$$

where $\mathcal{G}_{v}$ is the Mittag-Leffler function which is defined by

$$
\mathcal{G}_{v}\left(\frac{-v(\mathrm{t}-v)^{v}}{1-v}\right)=\sum_{n=0}^{\infty} \frac{\left(\frac{-v}{1-v}\right)^{n}(t-x)^{v n}}{\Gamma(v n+1)},
$$

and $\mathcal{B}(v)$ being a normalisation function.

Thus

$$
\begin{gathered}
\mathrm{ABR}_{\mathcal{D}_{a+}^{v}} \mathcal{F}(x)= \\
\frac{\mathcal{B}(v)}{1-v} \sum_{n=0}^{\infty}\left(\frac{-v}{1-v}\right)^{n}{ }^{R L} \mathcal{J}_{a}^{v n} \mathcal{F}(x),
\end{gathered}
$$

leads to $W(x, t)=u(\zeta)$,

$$
\begin{gathered}
\zeta=(1-v)\left(x^{-v n}+\right. \\
\left.k t^{-v n}\right)(B(v))^{-1}\left(\sum_{n=0}^{\infty}\left(-\frac{v}{1-v}\right)^{n} \Gamma(1-v n)\right)^{-1}
\end{gathered}
$$

where $k$ is the speed of the traveling wave.

Using wave transformation given in (5), Eq. (1) can be converted to ordinary differential equation (ODE). Twice integration of the obtained ODE with zero constant of the integration, gives

$$
\begin{gathered}
\left(k^{2}-\lambda^{2}\right) u(\zeta)-\alpha k^{2}(u(\zeta))^{2}+ \\
\sigma k^{2}(u(\zeta))^{3}-1 / 12 \lambda^{4} \frac{d^{2}}{d \zeta^{2}} u(\zeta)=0 .
\end{gathered}
$$

In the next sections, we will examine an ordinary differential equation (ODE) obtained above. The remainder of this paper is divided into five sections. In Sects. 2 and 3, methods are described briefly. In Sects. 4 and 5, proposed methods are applied to the model equation. In Sect. 6, results and discussions are given. In Sect. 7, conclusions and recommendations for future study are presented.

\section{The improved $\tan (\varphi / 2)$-expansion method}

In this section, the mathematical architecture (Manafian et al. 2016) is used to product exact traveling wave solutions. Consider the general nonlinear partial differential equation (NLPDE) for $q(x, t)$ is given by,

$$
P\left(q, q_{x}, q_{t}, q_{x x}, q_{t t}, \ldots\right)=0 .
$$


Taking into account wave transformation $\zeta=k x+$ $v t$, one can gain

$$
Q\left(q, k q^{\prime}, v q^{\prime}, k^{2} q^{\prime \prime}, v^{2} q^{\prime \prime}, \ldots\right)=0
$$

The solution of Eq. (8) can be articulated as

$$
q(\zeta)=S(\varphi)=\sum_{j=-M}^{M} A_{j}[\rho+\tan (\varphi / 2)]^{j},
$$

where $A_{j}(0 \leq j \leq M)$ and $A_{-j}=B_{j}(1 \leq j \leq M)$ are constants and $\rho$ is arbitrary constant, such that $A_{j} \neq 0, B_{j} \neq 0$ and $\varphi=\varphi(\zeta)$ is the solution of the following first order differential equation:

$$
\varphi^{\prime}(\zeta)=\gamma \sin (\varphi(\zeta))+\beta \cos (\varphi(\zeta))+\theta .
$$

If we try to find the solution of the (10), then we obtain special solutions that vary according to the state of the coefficients:

Family 1. When $\Delta=\gamma^{2}+\beta^{2}-\theta^{2}<0$ and $\beta-\theta \neq 0, \quad$ then $\quad \varphi(\zeta)=2 \tan ^{-1}\left[\frac{\gamma}{\beta-\theta}-\right.$ $\left.\frac{\sqrt{-\Delta}}{\beta-\theta} \tan \left(\frac{\sqrt{-\Delta}}{2} \bar{\zeta}\right)\right]$.

Family 2. When $\Delta=\gamma^{2}+\beta^{2}-\theta^{2}>0$ and $\beta-\theta \neq 0, \quad$ then $\quad \varphi(\zeta)=2 \tan ^{-1}\left[\frac{\gamma}{\beta-\theta}+\right.$ $\left.\frac{\sqrt{\Delta}}{\beta-\theta} \tanh \left(\frac{\sqrt{\Delta}}{2} \bar{\zeta}\right)\right]$.

Family 3. When $\Delta=\gamma^{2}+\beta^{2}-\theta^{2}>0$, $\beta \neq 0$ and $\theta=0$, then $\varphi(\zeta)=2 \tan ^{-1}\left[\frac{\gamma}{\beta}+\right.$ $\left.\frac{\sqrt{\beta^{2}+\gamma^{2}}}{\beta} \tanh \left(\frac{\sqrt{\beta^{2}+\gamma^{2}}}{2} \bar{\zeta}\right)\right]$.

Family 4. When $\Delta=\gamma^{2}+\beta^{2}-\theta^{2}<0$, $\theta \neq 0$ and $\beta=0$, then $\varphi(\zeta)=2 \tan ^{-1}\left[\frac{-\gamma}{\theta}+\right.$ $\left.\frac{\sqrt{\theta^{2}-\gamma^{2}}}{\theta} \tan \left(\frac{\sqrt{\theta^{2}-\gamma^{2}}}{2} \bar{\zeta}\right)\right]$.

Family 5. When $\Delta=\gamma^{2}+\beta^{2}-\theta^{2}>0$, $\beta-\theta \neq 0$ and $\gamma=0$, then $\varphi(\zeta)=$ $2 \tan ^{-1}\left[\sqrt{\frac{\beta+\theta}{\beta-\theta}} \tanh \left(\frac{\sqrt{\beta^{2}-\theta^{2}}}{2} \bar{\zeta}\right)\right]$.

Family 6. When $\gamma=0$ and $\theta=0$, then $\varphi(\zeta)=\tan ^{-1}\left[\frac{e^{2 \beta \bar{\zeta}}-1}{e^{2 \beta \bar{\zeta}}+1}, \frac{e^{2 \beta \bar{\zeta}}}{e^{2 \beta \bar{\zeta}}+1}\right]$.
Family 7. When $\beta=0$ and $\theta=0$, then $\varphi(\zeta)=\tan ^{-1}\left[\frac{e^{2 \gamma \bar{\zeta}}}{e^{2 \gamma \bar{\zeta}}+1}, \frac{e^{2 \gamma \bar{\zeta}}-1}{e^{2 \gamma \bar{\zeta}}+1}\right]$.

Family 8. When $\gamma^{2}+\beta^{2}=\theta^{2}$, then $\varphi(\zeta)=$ $2 \tan ^{-1}\left[\frac{\gamma \bar{\zeta}+2}{(\beta-\theta) \bar{\zeta}}\right]$.

Family 9. When $\gamma=\beta=\theta=r \gamma$, then $\varphi(\zeta)=2 \tan ^{-1}\left[e^{r \gamma \bar{\zeta}}-1\right]$.

Family 10. When $\gamma=\theta=r \gamma$ and $\beta=-r \gamma$, then $\varphi(\zeta)=-2 \tan ^{-1}\left[\frac{e^{r \gamma \bar{\zeta}}}{e^{r \gamma \bar{\zeta}}-1}\right]$.

Family 11. When $\theta=\gamma$, then $\varphi(\zeta)=$ $-2 \tan ^{-1}\left[\frac{(\gamma+\beta) e^{\beta \bar{\zeta}}-1}{(\gamma-\beta) e^{\beta \bar{\zeta}}-1}\right]$.

Family 12. When $\gamma=\theta$, then $\varphi(\zeta)=$ $2 \tan ^{-1}\left[\frac{(\theta+\beta) e^{\beta \bar{\zeta}_{1}}+1}{(\beta-\theta) e^{\beta \bar{\zeta}}-1}\right]$.

Family 13. When $\theta=-\gamma$, then $\varphi(\zeta)=$ $2 \tan ^{-1}\left[\frac{e^{\beta \bar{\zeta}}+\beta-\gamma}{e^{\beta \bar{\zeta}}-\beta-\gamma}\right]$.

Family 14. When $\beta=-\theta$, then $\varphi(\zeta)=$ $2 \tan ^{-1}\left[\frac{\gamma e^{\gamma \bar{\zeta}}}{1-\operatorname{de} \gamma \bar{\zeta}}\right]$.

Family 15. When $\beta=0$ and $\gamma=\theta$, then $\varphi(\zeta)=-2 \tan ^{-1}\left[\frac{\theta \bar{\zeta}+2}{\theta \bar{\zeta}}\right]$.

Family 16 . When $\gamma=0$ and $\beta=\theta$, then $\varphi(\zeta)=2 \tan ^{-1}[\theta \bar{\zeta}]$.

Family 17. When $\gamma=0$ and $\beta=-\theta$, then $\varphi(\zeta)=-2 \tan ^{-1}\left[\frac{1}{\theta \bar{\zeta}}\right]$.

Family 18. When $\gamma=0$ and $\beta=0$, then $\varphi(\zeta)=\theta \zeta+C$.

Family 19. When $\beta=\theta$, then $\varphi(\zeta)=$ $2 \tan ^{-1}\left[\frac{e^{\gamma \bar{\zeta}}-\theta}{\gamma}\right]$, where $\bar{\zeta}=\zeta+C, \rho, A_{0}, A_{i}, B_{i}(i=$ $1,2, \ldots, M), \gamma, \beta$ and $\theta$ are constants to be determined later.

As is often done in similar methods, balancing the highest order derivatives with the highest order nonlinear terms in Eq. (8), one can acquire $j$. Following determining $j$, if Eq. (9) is substitued into 
reduced equation (8), an algebraic equations set which contains $\tan (\varphi / 2)^{i}, \cot (\varphi / 2)^{i}, \quad(i=$ $0,1,2, \ldots)$ is obtained. Then setting each coefficient of $\tan (\varphi / 2)^{i}, \cot (\varphi / 2)^{i}$ to zero, we can get a set of over-determined equations for $A_{0}, A_{i}, B_{i}(i=$ $1,2, \ldots, M), \gamma, \beta, \theta$ and $\rho$. Using computer programming such as Maple, and Mathematica obtained system can be solved. Finally, $A_{0}, A_{1}, B_{1}, \ldots, A_{M}, B_{M}, \rho$ are replaced in the Eq. (9).

\section{The Simplest Equation Method}

In this section we will outline the simplest equation method proposed by Kudryashov (Kudryashov 2005, Kudryashov 2005) in 2005. Consider a NLPDE given in Eq. (7) and (8). Suppose that Eq. (8) have solutions in the following form:

$$
q(\zeta)=\sum_{i=0}^{M} A_{i}(P(\zeta))^{i},
$$

where $P(\zeta)$ satisfies the well-known Bernoulli and Riccati equations. Using of the balancing principle, the number $M$ can be calculated here. The coefficients $A_{0}, A_{1}, \ldots, A_{M}$ are constants.

If these well-known equations are used respectively, the form the solutions will have is given below.

\section{For the Bernoulli equation:}

$$
P^{\prime}(\zeta)=A P(\zeta)^{2}+B P(\zeta),
$$

where $A$ and $B$ are arbitrary constants. This equation is a well-known nonlinear ODE. The solution is represented as follows

$$
P(\zeta)=\frac{B(\cosh [B(\zeta+C)]+\sinh [B(\zeta+C)])}{1-A \cosh [B(\zeta+C)]-A \sinh [B(\zeta+C)]} .
$$

\section{For the Riccati equation}

$$
P^{\prime}(\zeta)=A P(\zeta)^{2}+B P(\zeta)+D,
$$

the solutions are represented as follows

$$
P(\zeta)=-\frac{B+\theta \tanh \left(\frac{1}{2} \theta(\zeta+C)\right)}{2 A},
$$

and

$$
\begin{array}{r}
P(\zeta)=-\frac{B+\theta \tanh \left(\frac{1}{2} \theta \zeta\right)}{2 A} \\
+\frac{\operatorname{sech}\left(\frac{\theta}{2} \zeta\right)}{C \cosh \left(\frac{\theta}{2} \zeta\right)-\frac{2 A}{\theta} \sinh \left(\frac{\theta}{2} \zeta\right)},
\end{array}
$$

where $\theta^{2}=B^{2}-4 \mathrm{AD}>0$.

\section{Application of ITEM}

Now, the ITEM will be explained for constructing traveling wave solutions to Eq. (6). With the help of the balancing principle between the $\frac{d^{2}}{d \zeta^{2}} u(\zeta)$ and $u^{3}(\zeta)$ in Eq. (6), we obtain $3 M=M+2, M=1$. Therefore, Eq. (9) is given as

$$
u(\zeta)=A_{0}+A_{1} \tan \left(\frac{\varphi(\zeta)}{2}\right)+B_{1}\left(\tan \left(\frac{\varphi(\zeta)}{2}\right)\right)^{-1} \text {. }
$$

Imposing the above equation into (6) and collect all terms with the same order $\tan (\varphi(\zeta) / 2)$ together and comparing, we obtain a set of algebraic equations of $\gamma, \beta, \theta, A_{0}, A_{1}, B_{1}$ as

$$
\begin{gathered}
-\frac{1}{12} \lambda^{4} B_{1} \beta \theta-\frac{1}{24} \lambda^{4} B_{1} \theta^{2}-\frac{1}{24} \lambda^{4} B_{1} \beta^{2}+\sigma k^{2} B_{1}{ }^{3}=0, \\
-\alpha k^{2} B_{1}{ }^{2}+3 \sigma k^{2} A_{0} B_{1}{ }^{2}-\frac{1}{8} \lambda^{4} B_{1} \gamma \theta-\frac{1}{8} \lambda^{4} B_{1} \gamma \beta=0, \\
3 \sigma k^{2} A_{0}{ }^{2} B_{1}+3 \sigma k^{2} A_{1} B_{1}{ }^{2}-\frac{1}{24} \lambda^{4} B_{1} \theta^{2}-2 \alpha k^{2} A_{0} B_{1} \\
+\frac{1}{24} \lambda^{4} B_{1} \beta^{2}-\lambda^{2} B_{1}-\frac{1}{12} \lambda^{4} B_{1} \gamma^{2} \\
+k^{2} B_{1}=0,
\end{gathered}
$$

$$
\begin{aligned}
-\lambda^{2} A_{0}-\frac{1}{24} \lambda^{4} A_{1} \gamma & \theta+\frac{1}{24} \lambda^{4} B_{1} \gamma \beta-\frac{1}{24} \lambda^{4} B_{1} \gamma \theta \\
+ & \sigma k^{2} A_{0}{ }^{3}-\frac{1}{24} \lambda^{4} A_{1} \gamma \beta-\alpha k^{2} A_{0}{ }^{2} \\
& +k^{2} A_{0}+6 \sigma k^{2} A_{0} A_{1} B_{1} \\
& -2 \alpha k^{2} A_{1} B_{1}=0
\end{aligned}
$$

$$
\begin{aligned}
k^{2} A_{1}-\frac{1}{12} \lambda^{4} A_{1} \gamma^{2} & +3 \sigma k^{2} A_{1}^{2} B_{1}-\frac{1}{24} \lambda^{4} A_{1} \theta^{2}-\lambda^{2} A_{1} \\
& +\frac{1}{24} \lambda^{4} A_{1} \beta^{2}+3 \sigma k^{2} A_{0}^{2} A_{1} \\
& -2 \alpha k^{2} A_{0} A_{1}=0
\end{aligned}
$$

$$
\begin{gathered}
-\frac{1}{8} \lambda^{4} A_{1} \gamma \theta+3 \sigma k^{2} A_{0} A_{1}{ }^{2}+\frac{1}{8} \lambda^{4} A_{1} \gamma \beta-\alpha k^{2} A_{1}{ }^{2}=0, \\
\sigma k^{2} A_{1}{ }^{3}+\frac{1}{12} \lambda^{4} A_{1} \beta \theta-\frac{1}{24} \lambda^{4} A_{1} \beta^{2}-\frac{1}{24} \lambda^{4} A_{1} \theta^{2}=0 .
\end{gathered}
$$


Solving the above algebraic equations with the help of Maple, we get three sets of coefficients for the solutions.

\section{Case I}

We have the desired constants as

$k=\frac{\sqrt{36+3 \Delta \lambda^{2}} \lambda}{6}, \lambda=\lambda, \sigma=\frac{2\left(12+\Delta \lambda^{2}\right) \alpha^{2}}{9 \Delta \lambda^{2}}, A_{0}=$ $-\frac{3 \lambda^{2}(-\Delta+\gamma \sqrt{\Delta})}{2\left(12+\Delta \lambda^{2}\right) \alpha}, A_{1}=\frac{3 \sqrt{\Delta}(-\theta+\beta) \lambda^{2}}{2\left(12+\Delta \lambda^{2}\right) \alpha}, B_{1}=0$.

By using Family 1, (6) becomes

$$
u_{1}=-\frac{3}{2} \frac{\lambda^{2}\left(-\Delta+\sqrt{\Delta} \sqrt{-\Delta} \tan \left(\frac{1}{2} \sqrt{-\Delta} \zeta\right)\right)}{\alpha\left(\lambda^{2} \Delta+12\right)},
$$

where $\Delta=\beta^{2}+\gamma^{2}-\theta^{2}$ and $\zeta$ is given in (5).

By using Family 2, (6) becomes

$$
u_{2}=\frac{3}{2} \frac{\lambda^{2}\left(\Delta+\tanh \left(\frac{1}{2} \sqrt{\Delta} \zeta\right) \Delta\right)}{\alpha\left(\lambda^{2} \Delta+12\right)}
$$

where $\Delta=\beta^{2}+\gamma^{2}-\theta^{2}$ and $\zeta$ is given in (5).

By using Family 3, (6) one gets

$$
u_{3}=\frac{3}{2} \frac{\lambda^{2}\left(\beta^{3}+\beta \gamma^{2}+\left(\gamma^{2}+\beta^{2}\right) \beta \tanh \left(\frac{1}{2} \sqrt{\gamma^{2}+\beta^{2}} \zeta\right)\right)}{\beta \alpha\left(\lambda^{2}\left(\gamma^{2}+\beta^{2}\right)+12\right)},
$$

where $\zeta$ is given in (5).

By using Family 4, (6) can be written as

$$
u_{4}=\frac{3}{2} \frac{\lambda^{2}\left(\theta \gamma^{2}-\theta^{3}-i\left(\theta^{2}-\gamma^{2}\right) \theta \tan \left(\frac{1}{2} \sqrt{\theta^{2}-\gamma^{2}} \zeta\right)\right)}{\theta \alpha\left(\lambda^{2}\left(\gamma^{2}-\theta^{2}\right)+12\right)},
$$

where $\zeta$ is given in (5).

By using Family 5, (6) can be written as

$$
u_{5}=\frac{3}{2} \frac{\lambda^{2}(\beta-\theta)\left(\beta+\theta+|\beta+\theta| \tanh \left(\frac{1}{2} \sqrt{\beta^{2}-\theta^{2}} \zeta\right)\right)}{\alpha\left(\lambda^{2} \beta^{2}-\lambda^{2} \theta^{2}+12\right)},
$$

where $\zeta$ is given in (5).

By using Family 13, (6) can be written as

$$
u_{6}=\frac{3 \lambda^{2} \beta^{2} \mathrm{e}^{\beta \zeta}}{\left(\mathrm{e}^{\beta \zeta}-\beta-\gamma\right) \alpha\left(\lambda^{2} \beta^{2}+12\right)},
$$

where $\beta>0$ and $\zeta$ is given in (5).

By using Family 18, (6) can be written as

$$
u_{7}=\frac{3 \theta \lambda^{2}\left(\theta+\sqrt{-\theta^{2}} \tan \left(\frac{1}{2} \theta \zeta+\frac{1}{2} C\right)\right)}{2 \alpha\left(-12+\lambda^{2} \theta^{2}\right)},
$$

where $\zeta$ is given in (5) and $C$ is arbitrary constant.

\section{Case II}

We have the desired constants as

$$
\begin{gathered}
k=\frac{\sqrt{36+3 \Delta \lambda^{2}}}{6} \lambda, \lambda=\lambda, \sigma= \\
\frac{2\left(12+\Delta \lambda^{2}\right) \alpha^{2}}{9 \Delta \lambda^{2}}, A_{0}=\frac{3 \lambda^{2}(\Delta+\gamma \sqrt{\Delta})}{2\left(12+\Delta \lambda^{2}\right) \alpha}, A_{1}=0, B_{1}= \\
\frac{3 \sqrt{\Delta} \lambda^{2}(\theta+\beta)}{2\left(12+\Delta \lambda^{2}\right) \alpha^{\prime}}
\end{gathered}
$$

By using Family 1, (6) becomes

$u_{8}=\frac{3}{2} \frac{\lambda^{2}\left(-\gamma \Delta-\Delta \sqrt{\Delta}+\sqrt{-\Delta}(\Delta+\gamma \sqrt{\Delta}) \tan \left(\frac{1}{2} \sqrt{-\Delta} \zeta\right)\right)}{\left(\lambda^{2} \Delta+12\right) \alpha\left(-\gamma+\sqrt{-\Delta} \tan \left(\frac{1}{2} \sqrt{-\Delta} \zeta\right)\right)}$,

where $\zeta$ is given in (5) and $\Delta=\beta^{2}-\theta^{2}+\gamma^{2}$.

By using Family 2, (6) becomes

$u_{9}=\frac{3}{2} \frac{\lambda^{2} \Delta(\gamma+\sqrt{\Delta})\left(1+\tanh \left(\frac{1}{2} \sqrt{\Delta} \zeta\right)\right)}{\left(\lambda^{2} \Delta+12\right) \alpha\left(\gamma+\sqrt{\Delta} \tanh \left(\frac{1}{2} \sqrt{\Delta} \zeta\right)\right)}$

where $\zeta$ is given in (5) and $\Delta=\beta^{2}-\theta^{2}+\gamma^{2}$.

By using Family 3, (6) becomes

$$
\begin{gathered}
u_{10}= \\
\frac{3}{2} \frac{\lambda^{2}\left(\gamma^{2}+\beta^{2}\right)\left(\gamma+\sqrt{\gamma^{2}+\beta^{2}}\right)\left(\tanh \left(\frac{1}{2} \sqrt{\gamma^{2}+\beta^{2}} \zeta\right)+1\right)}{\alpha\left(\lambda^{2}\left(\beta^{2}+\gamma^{2}\right)+12\right)\left(\gamma+\sqrt{\gamma^{2}+\beta^{2}} \tanh \left(\frac{1}{2} \sqrt{\gamma^{2}+\beta^{2}} \zeta\right)\right)}
\end{gathered}
$$

where $\zeta$ is given in (5).

By using Family 4, (6) becomes

$u_{11}=\frac{3}{2} \frac{\lambda^{2}\left(-\Delta(\gamma+\sqrt{\Delta})+(\Delta+\gamma \sqrt{\Delta}) \sqrt{-\Delta} \tan \left(\frac{1}{2} \sqrt{-\Delta} \zeta\right)\right)}{\left(\lambda^{2} \Delta+12\right) \alpha\left(-\gamma+\sqrt{-\Delta} \tan \left(\frac{1}{2} \sqrt{-\Delta} \zeta\right)\right)}$,

where $\zeta$ is given in (5) and $\Delta=\gamma^{2}-\theta^{2}$.

By using Family 5, (6) becomes

$$
\begin{gathered}
u_{12}= \\
\frac{3}{2} \lambda^{2} \sqrt{\frac{\beta-\theta}{\beta+\theta}} \frac{\left(\sqrt{\Delta}(\beta+\theta)+\sqrt{\frac{\beta+\theta}{\beta-\theta}} \tanh \left(\frac{1}{2} \sqrt{\Delta} \zeta\right) \Delta\right)}{\left(\lambda^{2} \Delta+12\right) \alpha \tanh \left(\frac{1}{2} \sqrt{\Delta} \zeta\right)},
\end{gathered}
$$

where $\zeta$ is given in (5) and $\Delta=\beta^{2}-\theta^{2}$.

By using Family 13, (6) becomes

$$
u_{13}=\frac{3 \lambda^{2} \beta^{2} \mathrm{e}^{\beta \zeta}}{\left(\lambda^{2} \beta^{2}+12\right) \alpha\left(\mathrm{e}^{\beta \zeta}+\beta-\gamma\right)},
$$


where $\beta>0$ and $\zeta$ is given in (5).

By using Family 18, (6) becomes

$u_{14}=\frac{3 \lambda^{2} \theta\left(\tan \left(\frac{1}{2} \theta \zeta+\frac{1}{2} C\right) \theta-\sqrt{-\theta^{2}}\right)}{2\left(-12+\lambda^{2} \theta^{2}\right) \alpha \tan \left(\frac{1}{2} \theta \zeta+\frac{1}{2} C\right)}$

where $\zeta$ is given in (5).

By using Family 19, (6) becomes

$u_{15}=-\frac{3 \lambda^{2} \gamma^{2} \theta}{\left(\lambda^{2} \gamma^{2}+12\right) \alpha\left(\mathrm{e}^{\gamma \zeta}-\theta\right)^{\prime}}$

where $\gamma<0$ and $\zeta$ is given in (5).

\section{Case III}

We have the desired constants as

$$
\begin{array}{r}
k=\frac{\sqrt{36+3 \Delta \lambda^{2}} \lambda}{6}, \lambda=\lambda, \sigma= \\
\frac{2\left(12+\Delta \lambda^{2}\right) \alpha^{2}}{9 \lambda^{2} \gamma^{2}}, A_{0}=\frac{3 \lambda^{2} \gamma^{2}}{\left(12+\Delta \lambda^{2}\right) \alpha}, A_{1}= \\
-\frac{3 \lambda^{2} \gamma(-\theta+\beta)}{2\left(12+\Delta \lambda^{2}\right) \alpha}, B_{1}=\frac{3 \lambda^{2} \gamma(\theta+\beta)}{2\left(12+\Delta \lambda^{2}\right) \alpha} .
\end{array}
$$

By using Family 1, (6) becomes

$$
u_{16}=\frac{-3 \lambda^{2} \gamma\left(\Delta+\Delta\left(\tan \left(\frac{1}{2} \sqrt{-\Delta} \zeta\right)\right)^{2}\right)}{2\left(\lambda^{2} \Delta+12\right) \alpha\left(-\gamma+\sqrt{-\Delta} \tan \left(\frac{1}{2} \sqrt{-\Delta} \zeta\right)\right)^{\prime}}
$$

where $\zeta$ is given in (5) and $\Delta=\beta^{2}-\theta^{2}+\gamma^{2}$.

By using Family 2, (6) becomes

$$
u_{17}=\frac{-3 \lambda^{2} \Delta \gamma\left(-1+\left(\tanh \left(\frac{1}{2} \sqrt{\Delta \zeta}\right)\right)^{2}\right)}{2\left(\lambda^{2} \Delta+12\right) \alpha\left(\gamma+\sqrt{\Delta} \tanh \left(\frac{1}{2} \sqrt{\Delta} \zeta\right)\right)^{\prime}}
$$

where $\zeta$ is given in (5) and $\Delta=\beta^{2}-\theta^{2}+\gamma^{2}$. By using Family 3, (6) becomes

$$
\begin{aligned}
& u_{18}= \\
& \frac{3 \lambda^{2} \gamma \beta\left(\gamma^{2}+\beta^{2}\right)\left(1-\left(\tanh \left(\frac{1}{2} \sqrt{\gamma^{2}+\beta^{2}} \zeta\right)\right)^{2}\right)}{2\left(\lambda^{2}\left(\beta^{2}+\gamma^{2}\right)+12\right) \alpha \beta\left(\gamma+\sqrt{\gamma^{2}+\beta^{2}} \tanh \left(\frac{1}{2} \sqrt{\gamma^{2}+\beta^{2}} \zeta\right)\right)^{\prime}}
\end{aligned}
$$

where $\zeta$ is given in (5).

By using Family 4, (6) becomes

$u_{19}=\frac{3 \lambda^{2} \gamma\left(-\gamma^{2}+\theta^{2}\right) \theta\left(1+\left(\tan \left(\frac{1}{2} \sqrt{\theta^{2}-\gamma^{2}} \zeta\right)\right)^{2}\right)}{2\left(12+\lambda^{2}\left(\gamma^{2}-\theta^{2}\right)\right) \alpha \theta\left(-\gamma+\sqrt{\theta^{2}-\gamma^{2}} \tan \left(\frac{1}{2} \sqrt{\theta^{2}-\gamma^{2}} \zeta\right)\right)^{\prime}}$, where $\zeta$ is given in (5).

By using Family 8, (6) becomes

$u_{20}=\frac{-\gamma \lambda^{2}}{2 \alpha \zeta(\gamma \zeta+2)^{\prime}}$

where $\zeta$ is given in (5).

By using Family 13, (6) becomes

$u_{21}=\frac{-6 \lambda^{2} \gamma \mathrm{e}^{\beta \zeta} \beta^{2}}{\left(\lambda^{2} \beta^{2}+12\right) \alpha\left(\mathrm{e}^{\beta \zeta}-\beta-\gamma\right)\left(\mathrm{e}^{\beta \zeta}+\beta-\gamma\right)}$,

where $\beta>0$ and $\zeta$ is given in (5).

By using Family 15, (6) becomes

$u_{22}=-\frac{\theta \lambda^{2}}{2 \alpha \zeta(\theta \zeta+2)^{\prime}}$

where $\zeta$ is given in (5).

By using Family 19, (6) becomes

$u_{23}=\frac{3 \lambda^{2} \gamma^{2} \mathrm{e}^{\gamma \zeta}}{\left(\lambda^{2} \gamma^{2}+12\right) \alpha\left(\mathrm{e}^{\gamma \zeta}-\theta\right)^{\prime}}$

where $\zeta$ is given in (5) and $\gamma<0$.

\section{Application of Simplest Equation Method}

In this section, we consider (8). With the help of homogenous balance principle between the $\frac{d^{2}}{d \zeta^{2}} u(\zeta)$ and $u^{3}(\zeta)$ in Eq. (6), we obtain $3 M=M+$ $2, M=1$. Therefore, we get that the trial solution of Eq. (6) can be stated as,

$$
u(\zeta)=A_{0}+A_{1} w(\zeta),
$$

where $A_{1} \neq 0, A_{0}$ are constants. For Bernoulli equation, putting $u, u^{\prime \prime}, u^{2}$ in (6) and comparing, we get,

$$
\begin{gathered}
k^{2} A_{0}-\lambda^{2} A_{0}+\sigma k^{2} A_{0}{ }^{3}-\alpha k^{2} A_{0}{ }^{2}=0, \\
k^{2} A_{1}-2 \alpha k^{2} A_{0} A_{1}-\lambda^{2} A_{1}+3 \sigma k^{2} A_{0}{ }^{2} A_{1} \\
-1 / 12 \lambda^{4} A_{1} B^{2}=0, \\
\sigma k^{2}{A_{1}}^{3}-1 / 6 \lambda^{4} A_{1} A^{2}=0, \\
-\alpha k^{2}{A_{1}}^{2}+3 \sigma k^{2} A_{0} A_{1}{ }^{2}-1 /
\end{gathered}
$$

$4 \lambda^{4} A_{1} B A=0$.

Calculation with the aid of Maple software, the solutions of the algebraic equations can be derived. 


$$
\operatorname{Set1}_{\mathrm{B}}:\left\{\begin{array}{l}
A_{0}=0, A_{1}=-\frac{2}{3} \frac{A \alpha}{\sigma B}, \lambda=\frac{2 \sqrt{6} \alpha}{B \sqrt{9 \sigma-2 \alpha^{2}}}, \\
k=\frac{6 \sqrt{6} \sqrt{\sigma} \alpha}{\left(-9 \sigma+2 \alpha^{2}\right) \mathrm{B}} .
\end{array}\right\}
$$

For Set $1_{B}$, we obtained the desired solutions as

$u_{24}=\frac{\frac{2}{3} \frac{A \alpha \cosh (B(\zeta+C))}{\sigma}+\frac{2}{3} \frac{A \alpha \sinh (B(\zeta+C))}{\sigma}}{-1+A \cosh (B(\zeta+C))+A \sinh (B(\zeta+C))^{\prime}}$

where $\zeta$ is given in (5).

Set2 $_{\mathbf{B}}:\left\{\begin{array}{l}A_{0}=0, A_{1}=-\frac{2}{3} \frac{A \alpha}{\sigma B}, \lambda=-\frac{2 \sqrt{6} \alpha}{B \sqrt{9 \sigma-2 \alpha^{2}}}, \\ k=\frac{6 \sqrt{6} \sqrt{\sigma} \alpha}{\left(-9 \sigma+2 \alpha^{2}\right) \mathrm{B}} .\end{array}\right\}$

For Set $2_{B}$, we obtained the desired solutions as

$u_{25}=\frac{\frac{2}{3} \frac{A \alpha \cosh (B(\zeta+C))}{\sigma}+\frac{2}{3} \frac{A \alpha \sinh (B(\zeta+C))}{\sigma}}{-1+A \cosh (B(\zeta+C))+A \sinh (B(\zeta+C))^{\prime}}$

where $\zeta$ is given in (5).

$$
\text { Set3 }_{\mathbf{B}}:\left\{\begin{array}{l}
A_{0}=\frac{2}{3} \frac{\alpha}{\sigma}, A_{1}=\frac{2}{3} \frac{A \alpha}{\sigma B}, \lambda=\frac{2 \sqrt{6} \alpha}{B \sqrt{9 \sigma-2 \alpha^{2}}}, \\
k=\frac{6 \sqrt{6} \sqrt{\sigma} \alpha}{\left(-9 \sigma+2 \alpha^{2}\right) \mathrm{B}} .
\end{array}\right\}
$$

For Set $3_{B}$, we obtained the desired solutions as

$$
u_{26}=\frac{-2 \alpha}{3 \sigma(-1+A \cosh (B(\zeta+C))+A \sinh (B(\zeta+C)))^{\prime}},
$$

where $\zeta$ is given in (5).

Set4 $_{\mathbf{B}}:\left\{\begin{array}{l}A_{0}=\frac{2}{3} \frac{\alpha}{\sigma}, A_{1}=\frac{2}{3} \frac{A \alpha}{\sigma B}, \lambda=-\frac{2 \sqrt{6} \alpha}{B \sqrt{9 \sigma-2 \alpha^{2}}}, \\ k=\frac{6 \sqrt{6} \sqrt{\sigma} \alpha}{\left(-9 \sigma+2 \alpha^{2}\right) \mathrm{B}} .\end{array}\right\}$

For Set $4_{B}$, we obtained the desired solutions as

$u_{27}=\frac{2 \alpha}{3 \sigma(-1+A \cosh (B(\zeta+C))+A \sinh (B(\zeta+C)))^{\prime}}$,

where $\zeta$ is given in (5).

For Riccati equation, putting $u, u^{2}, u^{\prime \prime}, u^{3}$ in (6) and comparing, we get,

$12 k^{2} A_{0}-\lambda^{4} A_{1} B D-12 \lambda^{2} A_{0}+12 \sigma k^{2} A_{0}{ }^{3}-$

$12 \alpha k^{2} A_{0}^{2}=0$,

$12 k^{2} A_{1}-12 \lambda^{2} A_{1}+36 \sigma k^{2} A_{0}^{2} A_{1}-$

$24 \alpha k^{2} A_{0} A_{1}-\lambda^{4} A_{1} B^{2}-2 \lambda^{4} A_{1} A \mathrm{D}=0$,
$-12 \alpha k^{2} A_{1}{ }^{2}-3 \lambda^{4} A_{1} B A+36 \sigma k^{2} A_{0} A_{1}{ }^{2}=0$,

$12 \sigma k^{2} A_{1}{ }^{3}-2 \lambda^{4} A_{1} A^{2}=0$.

Calculation with the aid of Maple software, the solutions of the algebraic equations can be derived.

$\operatorname{Set1}_{\mathbf{R}}:\left\{\begin{array}{l}A_{0}=\frac{1}{2} \frac{\left(B+\sqrt{-4 A D+B^{2}}\right) A_{1}}{A}, A_{1}=A_{1}, \\ \alpha=3 \frac{A}{k^{2} A_{1}} \frac{\sqrt{-4 A D+B^{2}}}{4 A D-B^{2}}\left(2 \frac{3+\sqrt{9-12 k^{2} A D+3 k^{2} B^{2}}}{4 A D-B^{2}}-k^{2}\right), \\ \lambda=\frac{\sqrt{2} \sqrt{\left(4 A D-B^{2}\right)\left(3+\sqrt{9-12 k^{2} A D+3 k^{2} B^{2}}\right)}}{4 A D-B^{2}}, \\ \sigma=2 A^{2} \frac{\left(2 \frac{3+\sqrt{9-12 k^{2} A D+3 k^{2} B^{2}}}{4 A D-B^{2}}-k^{2}\right)}{\left(4 A D-B^{2}\right) k^{2} A_{1}{ }^{2}} .\end{array}\right\}$

For Set $1_{R}$, we obtained the desired solutions as

$u_{28}=-\frac{A_{1} \sqrt{-4 A D+B^{2}}\left(-1+\tanh \left(\frac{1}{2} \sqrt{-4 A D+B^{2}}(\zeta+C)\right)\right)}{2 A}$,

and

$u_{29}=\frac{1}{2} \frac{\left(B+\sqrt{B^{2}-4 A D}\right) A_{1}}{A}$

$+A_{1}\left(-\frac{1}{2} \frac{B+\sqrt{B^{2}-4 A D} \tanh \left(\frac{1}{2} \sqrt{B^{2}-4 A D} \zeta\right)}{A}\right.$

$$
\left.+\frac{\operatorname{sech}\left(\frac{1}{2} \sqrt{B^{2}-4 A D} \zeta\right)}{C \cosh \left(\frac{1}{2} \sqrt{B^{2}-4 A D \zeta}\right)-2 \frac{A \sinh \left(\frac{1}{2} \sqrt{B^{2}-4 A D} \zeta\right)}{\sqrt{B^{2}-4 A D}}}\right)
$$

where $\zeta$ is given in (5).

$$
\operatorname{Set}_{\mathbf{R}}:\left\{\begin{array}{l}
A_{0}=\frac{1}{2} \frac{\left(B+\sqrt{-4 A D+B^{2}}\right) A_{1}}{A}, A_{1}=A_{1}, \\
\left.\alpha=\frac{3 A \sqrt{-4 A D+B^{2}}\left(2 \frac{3+\sqrt{9-12 k^{2} A D+3 k^{2} B^{2}}}{4 A_{1}\left(4 A D-B^{2}\right) k^{2}}-B^{2}\right.}{4 A D}-k^{2}\right), \\
\lambda=-\frac{\sqrt{2} \sqrt{\left(4 A D-B^{2}\right)\left(3+\sqrt{9-12 k^{2} A D+3 k^{2} B^{2}}\right)}}{4 A D-B^{2}}, \\
\sigma=\frac{2 A^{2}\left(2 \frac{3+\sqrt{9-12 k^{2} A D+3 k^{2} B^{2}}}{4 A D-B^{2}}-k\right)}{\left(4 A D-B^{2}\right) k^{2} A_{1}{ }^{2}} .
\end{array}\right\}
$$

For Set $2_{R}$, we obtained the desired solutions as

$$
u_{30}=\frac{\left(B+\sqrt{-4 A D+B^{2}}\right) A_{1}}{2 A}
$$

$-\frac{B A_{1}+A_{1} \sqrt{-4 A D+B^{2}} \tanh \left(\frac{1}{2} \sqrt{-4 A D+B^{2}}(\zeta+C)\right)}{2 A}$, 
and

$u_{31}$

$=\frac{1}{2} \frac{\left(B+\sqrt{-4 A D+B^{2}}\right) A_{1}}{A}$

$+A_{1}\left(-\frac{1}{2} \frac{B+\sqrt{-4 A D+B^{2}} \tanh \left(\frac{1}{2} \sqrt{-4 A D+B^{2}} \zeta\right)}{A}\right.$

$\left.+\frac{\operatorname{sech}\left(\frac{1}{2} \sqrt{-4 A D+B^{2}} \zeta\right)}{C \cosh \left(\frac{1}{2} \sqrt{-4 A D+B^{2}} \zeta\right)-2 \frac{A \sinh \left(\frac{1}{2} \sqrt{-4 A D+B^{2}} \zeta\right)}{\sqrt{-4 A D+B^{2}}}}\right)$

where $\zeta$ is given in (5).

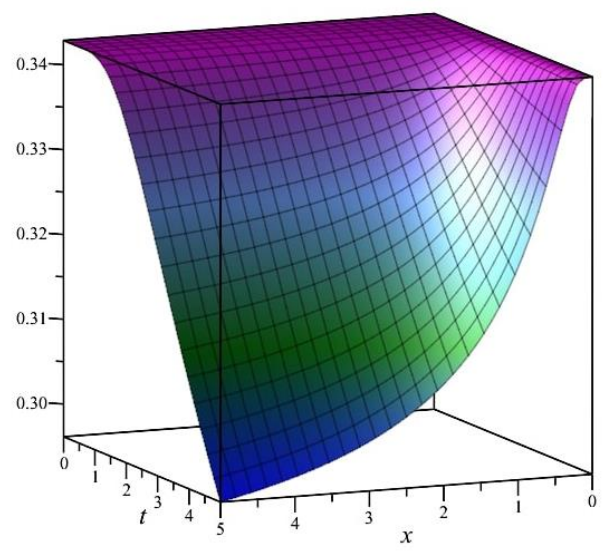

Figure 1. Numerical simulation of $u_{1}$ in Eq. (15) in three dimensional plot.

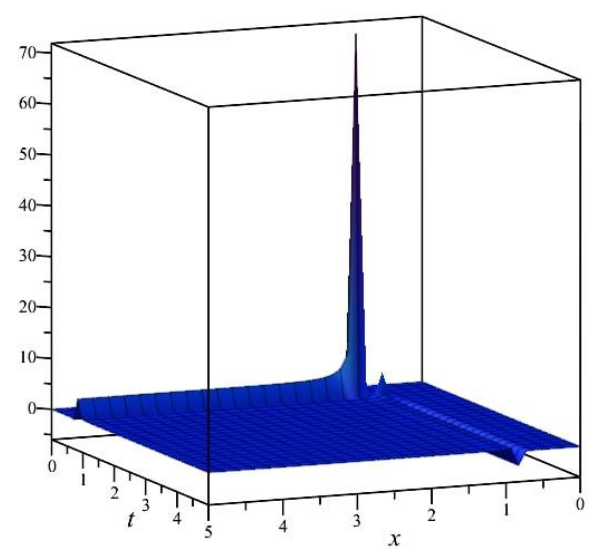

Figure 2. Numerical simulation of $u_{18}$ in Eq. (34) in three dimensional plot.

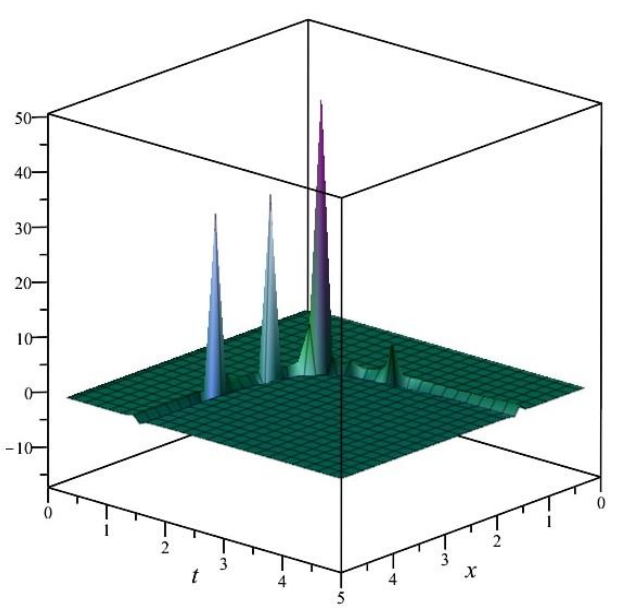

Figure 3. Numerical simulation of $u_{24}$ in Eq. (42) in three dimensional plot.

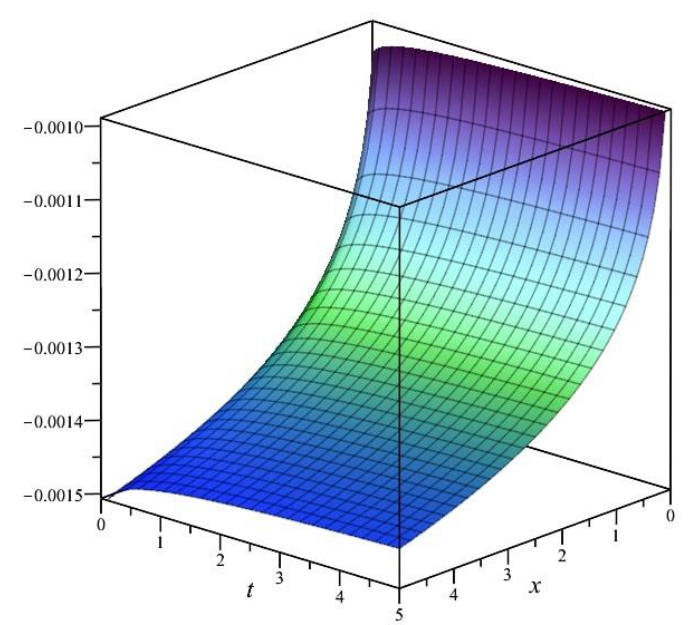

Figure 4. Numerical simulation of $u_{26}$ in Eq. (44) in three dimensional plot.

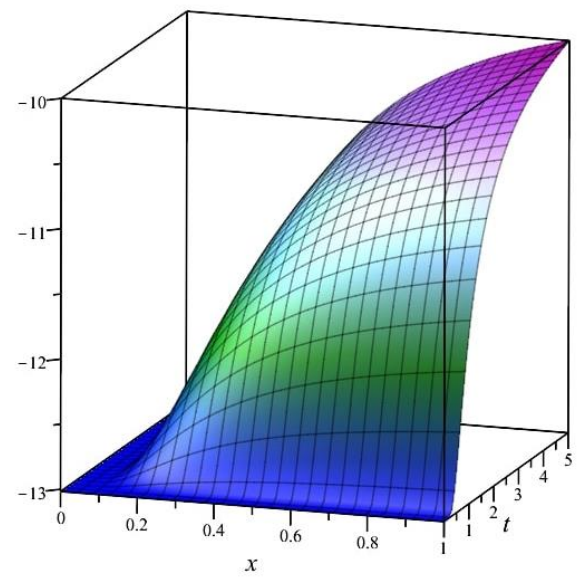

Figure 5. Numerical simulation of $u_{28}$ in Eq. (47) in three dimensional plot. 


\section{Results and discussion}

The model discussed in this study contains the fractional derivative $A B R$ which makes it interesting. The ABR fractional operator is considered as one of the last general fractional operators derived from avoiding the deficiencies and defects of some other fractional operators (Park et al. 2020). The techniques applied in this study are effective and powerful techniques for solving partial differential equations. The variety of solutions obtained makes these methods advantageous, which is a scientifically beatiful feature.

The nonlinear integer order PDE describing the lowpass electrical lines has been discussed in (Abdoulkary et al. 2013) using an auxiliary equation method an in (Zayed and Alurrfi 2015) using Jacobi elliptic function expansion method. In (Park et al. 2020), the fractional nonlinear model of the lowpass electrical transmission lines has been considered and constructed explicit wave solutions using modified Khater method. When our results obtained in this study using two different methods were compared with the results obtained in (Part et al 2020), it was seen that they were different.

The graphical representation of some of obtained solution are plotted by taking suitable values of involved unknown parameters. Here, we give the figure interpretation of the shown figures as following:

Figure 1 explains the periodic wave solution $\mathrm{u}_{1}$ when $a=1, b=1, c=2, \alpha=5, \lambda=4, v=0.1$.

Figure 2 shows the dark wave solution $\mathrm{u}_{18}$ when $a=2, b=5, c=0, \alpha=2, \lambda=0.6, v=0.55$.

Figure 3 shows the graph of hyperbolic function solution $\mathrm{u}_{24}$ when $A=3, B=2, C=2, \alpha=$ $-2, \sigma=5, v=0.3$.

Figure 4 shows the graph of hyperbolic function solution $\mathrm{u}_{26}$ when $A=1, B=1, C=2, \alpha=$ $0.1, \sigma=3, v=0.01$.

Figure 5 indicates the exact soliton wave solution $\mathrm{u}_{28} \quad$ when $A=-3, A_{1}=5, B=1, C=$ $-0.1, D=5, k=4.5, v=0.6$.

\section{Conclusions}

In this work, the improved $\tan (\varphi / 2)$-expansion method and the simple equation method are successfully used to obtained exact solutions of the fractional nonlinear model of the low-pass electrical transmission lines. By proposed methods, solitary solutions are established including three types namely, triangular functions solutions, exponential solutions and rational solutions. The variety of complete solutions obtained plays an important role in the interpretation and understanding of the physical model. Some sketches are also depicted for the interpretation physically of the achieved solutions. To our best knowledge, some of the obtained solutions are new and not reported previously. This study shows that the proposed method is quite proficient and practically well organized in finding exact solutions to other physical problems.

\section{References}

Abdoulkary, S., Beda, T., Dafounamssou, O., Tafo, E. W., Mohamadou, A., 2013. Dynamics of solitary pulses in the nonlinear low-pass electrical transmission lines through the auxiliary equation method. J. Mod. Phys. Appl., 2, 69-87.

Abdou, M.A. and Soliman, A.A., 2018. New exact travelling wave solutions for space-time fractional nonlinear equations describing nonlinear transmission lines. Results in Physics, 9, 1497-1501.

Alderremy, A. A., Attia, R. A., Alzaidi, J. F., Lu, D., Khater, M., 2019. Analytical and semi-analytical wave solutions for longitudinal wave equation via modified auxiliary equation method and Adomian decomposition method. Thermal Science, 00, 355355.

Atangana, A., Baleanu, D., 2016. New fractional derivatives with nonlocal and nonsingular kernel: Theory and application to heat transfer model. Thermal Science, 20, 2, 763- 769.

Atangana, A., Gomez-Aguilar, J.F. , 2018. Numerical approximation of Riemann-Liouville definition of fractional derivative: from Riemann-Liouville to Atangana-Baleanu. Numerical Methods for Partial Differential Equations, 34, 5, 1502-1523.

Atangana, A. And Koca, İ., 2016. Chaos in a simple nonlinear system with Atangana-Baleanu derivatives with fractional order. Chaos Solitons Fractals 89, 447-454.

Caputo, M. , 1967. Linear models of dissipation whose q is almost frequency independentâ' ii. Geophysical Journal International, 13, 5, 529-539.

Caputo, M. and Fabrizio, M., 2015. A new definition of fractional derivative without singular kernel. 
Progress in Fractional Differentiation and Applications, 1, 2,1-13.

Durur, H., 2020. Different types analytic solutions of the (1+ 1)-dimensional resonant nonlinear Schrödinger's equation using $\left(\mathrm{G}^{\prime} / \mathrm{G}\right)$-expansion method. Modern Physics Letters B, 34, 03, 2050036.

Durur, H., Yokuş, A., 2019. (1/G')-Açılım Metodunu Kullanarak Sawada-Kotera Denkleminin Hiperbolik Yürüyen Dalga Çözümleri. Afyon Kocatepe Üniversitesi Fen ve Mühendislik Bilimleri Dergisi, 19, 3, 615-619.

Durur, H., Yokuş, A., 2020. Analytical solutions of Kolmogorov-Petrovskii-Piskunov equation. Balıkesir Üniversitesi Fen Bilimleri Enstitüsü Dergisi, 22, 2, 628636.

Durur, H., Ilhan, E., Bulut, H., 2020. Novel complex wave solutions of the $(2+1)$-dimensional hyperbolic nonlinear Schrödinger equation. Fractal and Fractional, 4, 3, 41.

Fernandez, A., Özarslan, M.A. and Baleanu, D., 2019. On fractional calculus with general analytic kernels. Applied Mathematics and Computation, 354, 248265.

Khater, M. M., Ghanbari, B., Nisar, K. S., Kumar, D., 2020. Novel exact solutions of the fractional Bogoyavlensky-Konopelchenko equation involving the Atangana-Baleanu-Riemann derivative. Alexandria Engineering Journal, 59, 5, 2957-2967.

Kudryashov, N. A., 2005. Simplest equation method to look for exact solutions of nonlinear differential equations. Chaos Solitons and Fractals, 24, $1217-$ 1231.

Kudryashov, N. A., 2005. Exact solitary waves of the Fisher equation. Physics Letters A, 342, 99-106.

Manafian, J., Lakestani, M., and Bekir, A., 2016. Study of the analytical treatment of the $(2+1)$-dimensional Zoomeron, the Duffing and the SRLW equations via a new analytical approach. International Journal of Applied and Computational Mathematics, 2, 2, 243268.

Morales-Delgado, V.F., Gomez-Aguilar, J.F., TanecoHernandez, M. A., and Baleanu, D., 2018. Modeling the fractional non-linear Schrödinger equation via Liouville-Caputo fractional derivative. Optik, 162, 1-7.

Özpınar, F., 2020. Kesirli Mertebe Kısmi Diferensiyel Denklemlerin Ayrık Homotopi Perturbasyon Metodu ile Çözümü. Afyon Kocatepe Üniversitesi Fen Ve Mühendislik Bilimleri Dergisi, 20, 2, 213-221.
Park, C., Khater, M. M., Abdel-Aty, A. H., Attia, R. A., Rezazadeh, H., Zidan, A. M., Mohamed, A. B. 2020. Dynamical analysis of the nonlinear complex fractional emerging telecommunication model with higher-order dispersive cubic-quintic. Alexandria Engineering Journal, 59, 3, 1425-1433.

Podlubny I., 1999. Fractional differential equations. Academic Press, San Diego.

Rezazadeh, H., Khodadad, F. S. and Manafian, J., 2017. New structure for exact solutions of nonlinear time fractional Sharma-Tasso-Olver equation via conformable fractional derivative. Applications and Applied Mathematics: An International Journal, 12, 1, 13-21.

Ray, S.S., 2006. Exact solutions for time-fractional diffusion-wave equations by decomposition method. Physica Scripta, 75, 1, 53.

Shang, N., Zheng, B., 2013. Exact solutions for three fractional partial differential equations by the $\left(G^{\prime} / G\right)$ method. International Journal of Applied Mathematics, 43, 3, 114-119.

Tasbozan, O., Kurt, A., Durur, H., 2019. Implementation of new sub equation method to time fractional partial differential equations. International Journal of Engineering Mathematics and Physics, 1, 1-12.

Yasar, E., Yıldırım, Y. , 2018. On the Lie symmetry analysis and traveling wave solutions of time fractional fifth-order modified Sawada-Kotera equation. Karaelmas Science and Engineering Journal, 8, 2, 411-416.

Yokuş, A., 2020. On the exact and numerical solutions to the FitzHugh-Nagumo equation. International Journal of Modern Physics B, 34, 17, 2050149.

Yokus, A., Durur, H., Ahmad, H., Yao, S. W., 2020. Construction of different types analytic solutions for the Zhiber-Shabat equation. Mathematics, 8, 6, 908.

Yokus, A., Durur, H., Ahmad, H., 2020. Hyperbolic type solutions for the couple Boiti-Leon-Pempinelli system. Facta Universitatis, Series: Mathematics and Informatics, 35, 2, 523-531.

Yokuş, A., Durur, H., Abro, K. A., Kaya, D., 2020. Role of Gilson-Pickering equation for the different types of soliton solutions: a nonlinear analysis. The European Physical Journal Plus, 135, 8, 1-19.

Yokuş, A., Durur, H., Nofal, T. A., Abu-Zinadah, H., Tuz, M., Ahmad, H., 2020. Study on the applications of two analytical methods for the construction of traveling wave solutions of the modified equal width equation. Open Physics, 18, 1, 1003-1010. 
Zayed, E. M. E., Alurrfi, K. A. E., 2015. A new Jacobi elliptic function expansion method for solving a nonlinear PDE describing the nonlinear low-pass electrical lines. Chaos, Solitons, Fractals, 78, 148-155. 